\title{
Green Computing Under Cloud Environment Proposed architecture using cloud computing \& thin client
}

\author{
${ }^{1}$ T.Chandrasekar, ${ }^{2}$ K.Senthil Kumar \\ ${ }^{1,2} P G$ Scholar, Department of Computer Science \& EngineeringPaavai Engineering College Namakkal, India
}

\begin{abstract}
Private Cloud computing provides attractive \& cost efficient Server Based Computing (SBC). The implementation of Thin client computing for private cloud computing will reduce the IT Cost and consumes less power. Most cloud services run in browser based environment so we don't need a fat client to use in the private Cloud environment. Implementing Thin Client Technology along with Private Cloud Computing will help to reduce the IT Operational Cost by $90 \%$ by saving power, space and maintenance. It requires only minimal power for cooling the Infrastructure. Thin Client with private Cloud Computing can be referred as purest form of green computing \& carbon free computing.

Keywords: Thin Clients with Cloud computing; Green computing; Private Cloud Terminal Computing; Carbon Free Computing; Low Powered Computing.

\section{Introduction}

Private Cloud Computing is the technology where all desktop computers can access the private cloud server for the purpose of processing client request \& data storage. Application and other services are offered under on demand basis. The virtualization technology enables multiple operating systems to be implemented on single computer or CPU. The virtualization Technology enables us to divide the physical hardware of a computer into many logical units and help to implement multiple operating systems into one physical hardware unit. Thin Client Technology makes use of server based computing. All the application are installed in Host pc and all the clients collects user input from client node and passes to host pc for computing. The host pc processes the request and sends the final output to the clients. By combining cloud computing, virtualization and thin client technology creates the low powered, low cost, low maintenance computing environment. We can call this environment as a pure green IT environment, Rural IT environment and Carbon free computing.
\end{abstract}

\section{Thin Client Technology}

A. Overview of Served Based Computing.

The Traditional Server based computing will collect the user inputs from client terminals and passed this input to the Host pc or server. The server will perform the required operation and passed the final output to the thin client terminal. The major drawback of traditional server based computing is bandwidth usage. Today's applications require huge amount of bandwidth transfer between client and server. Traditional method of Server based computing is practically not suitable for latest application. Then web based thin client technology is adapted but practically most commercial application not support web based architectures.

\section{B. Thin Client Technology -A solution to SBC Drawbacks}

The thin client technology is perfect solution to reduce the drawback of bandwidth used in server based computing. Thin Client technology runs the client application in terminal server of host pc or server and transmit only the screen images to the clients instead of sending the whole processed data's, reducing network traffic, avoiding installation of client software on a remote client or workstation and facilitating administration and deployment much better than the traditional server based computing.

In Thin Client Technology, a terminal server will be deployed in host pc or server and it will fully runs the client part of the application in Host pc or server itself. This technology uses a multi user operating system as a terminal server running multiple client sessions on a single server and a protocol that shows user Interface

in client terminals. 


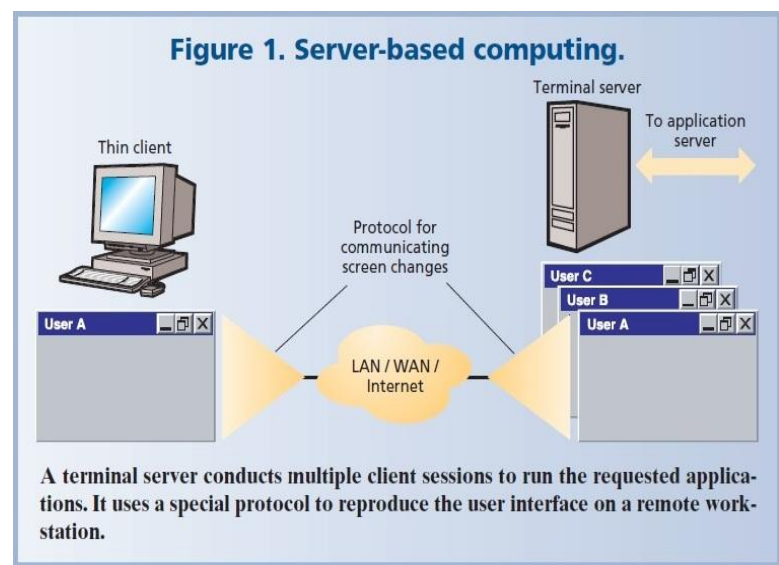

Figure 1 shows this architecture. The only application present inside the client terminal is the application which reproduces the user interface received from terminal server. The thin client technology supports many client platforms with low band width usage. It supports any type of client server application.

Citrix modified Windows NT 3.5 and modified it as multiuser operating system capable of supporting thin client on special terminals. Microsoft and Citrix signed a joint agreement for using Citrix technology and this agreement is the basic step of development for Windows NT 4.0 terminal server edition. Citrix currently uses the own architecture called ICA(Independent Computer Architecture) for reproducing a user interface on a thin client terminal. The architecture transfers only the mouse clicks, keyboard inputs and screen refresh to the server enabling lower band width usage. It uses less than $20 \mathrm{Kbits} / \mathrm{sec}$. Citrix provides numerous tools for access management, resource and user management.

Microsoft also developed its own Remote Desktop protocol called RDP for connecting windows client to terminal servers. Citrix offered special tools for reproducing images to the client node includes meta frame, NFuse \& Extranet.

\section{Virtualization}

Virtualization is the process of creating virtual environment of some physical environment. The best example will be our hard disks in computers; we are dividing physical hard disks into multiple logical drives.

\section{A. Hardware Virtualization:}

Hardware virtualization is the process of creating virtual machines that will perform like a normal computer with operating system. Here the bare hardware is divided into multiple logical units like our hard disks and we are implementing multiple operating systems into the logical hardware units and making all together to run in one time. A single Computer now can run multiple operating systems at the same time using virtualization concept. Software executed on these machines is separated from the underlying hardware resources. The machine which runs windows Xp can host a virtual machine that runs the Ubuntu operating system. The Ubuntu software is run on Ubuntu operating system using the same hardware resources that runs windows XP.

In virtualization the host $\mathrm{PC}$ is a bare hardware where the visualization takes places and guest pc is a virtual machine created by using host PC. The word host and guest are used to distinguish the software runs on physical machine and software runs on virtual machine.

The software that creates a virtual machine is also called as the virtual machine manager. Examples are hypervisor or VM Ware Vsphere. This software creates a multiple logical hardware units on the bare hardware. Logical units are called as virtual machines and physical units are called as bare hardware.

\section{B. Snapshots}

A snapshot is a state of virtual machine in particular time. This is mainly mentioned in storage disks. The snapshot can be taken at given time interval and it can be reverted back on any time. A Snapshot is a point in time image of the virtual guest operating system (VM). The snapshot contains the image of the virtual disk, RAM and device drivers at the time of the snapshot are taken.

\section{Teleportation}

The snapshot mentioned above can be moved to the new host pc using its own hypervisor. If the VM is stopped, snapshotted moved and resumed on the new host pc. This process is called migration. If the snapshots are taken periodically then this process is very fast and it can be migrated to the new host pc without disturbing the service and we can take the old host pc for physical maintenance. 


\section{Failover}

Failover is the process of switching VM images or VM machines to one host pc to another host pc in case of abnormal failures in present host pc.

\section{E. Challenges.}

The main issue faced in virtualization is licensing of proprietary software. If a physical Linux Server OS provided virtualized windows operating system environment then still it require a windows license requirement for running this windows operating system in Host pc. This kind of issues affects the on demand virtualization services because the demand is unpredicted and it's not possible to purchase the huge no of licenses in advanced. Today most application vendors are created new license agreement that will be adopted to virtualization environment.

\section{Private Cloud Computing.}

Private Cloud refers the IT Infrastructure which runs its application software in hosted environment. The private cloud is implemented within the organization behind the firewall. The private cloud is hosted internally or externally. Even ifs it's hosted externally still it's accessed within the organization. The drawback of private cloud computing is its initial cost of set up. The initial cost of set up includes setting up of client terminals to access the private cloud and its implementation cost which includes mapping proprietary software into hosted private cloud computing environment.

The running cost of private cloud is practically unpredictable because of its changing nature. But the cost will be comparatively lower then public cloud because of low usage of Internet bandwidth. In private cloud we can customize the infrastructure environment as per our needs, but in public cloud we are not able to customize the infrastructure and it's a pre-defined suite of a public cloud vendor.

The private cloud computing environment is secured because the internal data will not be accessed by outside the cloud. The data security mechanism can also be additionally implemented to protect our data. Since it's a hosted service mostly data security task will be implemented by private cloud computing suites.

We can scale up the private cloud computing infrastructure easily by adding additional client terminals. Updating Client is also simple task because any private cloud computing environment just requires a browser enabled operating system; it can be updated easily by remote firmware update.

\section{Purpose Of Proposed System}

The proposed system is designed to provide low cost computing with zero maintenance in client terminal. Today computing user is varying from educated people to uneducated people who use computers to finish the task. Assume the person in billing terminal of point of sale system is not aware about the hardware or software maintenance in client node. These kinds of users want a trouble free environment. The proposed system gives zero maintenance environments.

Proposed Terminal consumes less power compared to traditional fat client and cost of client terminal is also much lesser then cost of fat clients. The proposed system will help to build a computing environment for rural people in government schools with minimum infrastructure set up cost and operating cost. The electricity will be saved using proposed system.

Proposed system prevents from E Waste, The up gradation in physical server helps to run all client nodes as an updated server, so no need to change the client terminal often. The fat client requires up gradation often.

\section{A. Low Power Consumption}

\section{Advantages Of Proposed System}

Proposed Client terminal runs using A9 processor and $512 \mathrm{MB}$ of RAM which require only $5 \mathrm{~W}$ of power, The A9 processor is thermally constrained processor which runs without a fan, this fan less design assures proposed client terminal generates less heat. In physical server we are virtualizing to make use of single physical server to run as two virtual machine which additionally enables more power saving. Both operating system runs using the single physical server.

The following tables show the power consumption of CPU and proposed system 
TABLE 1

\begin{tabular}{|l|l|}
\hline CPU & Proposed System \\
\hline 70W (for Core i3 Processor) & 5W(for ARM A9 Processor) \\
\hline
\end{tabular}

\section{B. Less $E$ Waste}

Proposed system uses hosted cloud server to run its application via web browser. The private cloud server hardware update enables all client nodes to be work like new updated server. The traditional fat client requires frequent changing of its hardware depending upon the advancement in technology. More no of moving parts, there will be a huge chance for failure in hardware components. Proposed system consists of no moving parts and most components are embedded into a single board which makes the chance of failure lesser then the traditional system.

\section{Low Maintainance}

Our proposed system consists of embedded operating system and remote firm ware support and all the services are provided using web browser which eliminates the requirement of hardware or network engineer on site. The remote firmware update option enables to update the firm ware from remote location.

\section{Requires minimal cost for licensing.}

The cost of terminal server and client access license is lower than the cost of traditional standalone operating system. The simple web browser can be implemented in real operating system such as android or linux which enables absolutely no license required in client side and in terminal server we require only client access license. If we go for private cloud then there is no need for the client access license in our proposed terminal, which reduces overall cost of the IT infrastructure.

\section{Proposed System}

In proposed architecture, the fat client is replaced by low powered terminal device with embedded operating system that supports web browser and RDP protocols. The cloud server is virtualized into two virtual machines. Two different server operating systems can be loaded into the two virtual machines and we are hosting applications in both virtual machines. With the help of Virtualization software both operating system is executed simultaneously. Hosting is done in both operating systems now low powered access terminal with browser can access any hosted services and run like normal traditional computer. Figure 2 shows the architecture of the system.

Figure 2

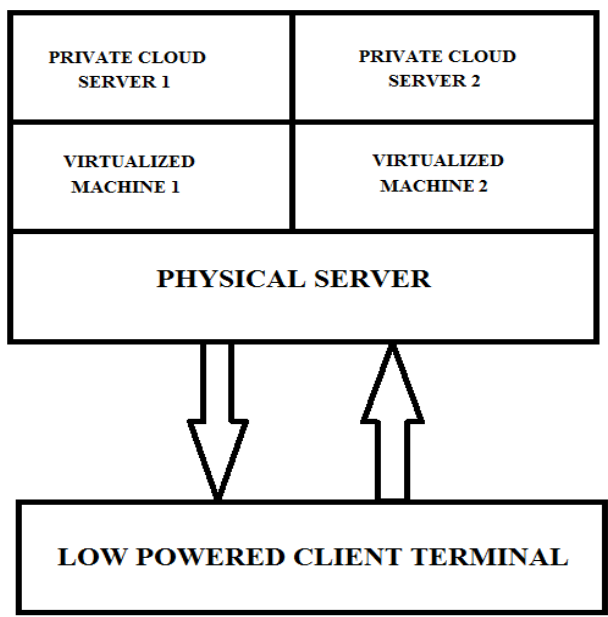

\section{A. Low Powered Client Terminal.}

The low powered client terminal is a device which contains minimal set of hardware components to support operating system like android or windows CE with browser support. The browser will be used to access the hosted service in private cloud. The additional RDP protocol is used to access the traditional terminal server. The additional traditional Server is used along with cloud server to provide additional services which is not provided by hosted private cloud. By implementing RDP 7.0 in low powered client terminal, multimedia contents can be played in client side. 
B. Specification of proposed Low powered Client terminal

\begin{tabular}{|c|c|}
\hline Component Type & Specification \\
\hline Processor & ARM-A9 \\
\hline Operating System & Android or Embedded Linux \\
\hline Ram & $512 \mathrm{MB}$ \\
\hline Flash Memory & $512 \mathrm{MB}$ \\
\hline Storage & $512 \mathrm{MB}$ DOM \\
\hline Power Consumption & $5 \mathrm{~W}$ \\
\hline Protocol & RDP 7.1, HTTP \\
\hline
\end{tabular}

\section{Benefit of low powered terminal devices.}

The low powered client terminal is designed using ARM - A9 processor which consumes less power comparing to traditional processor used in fat clients. Client Terminal requires only web browser and RDP protocol support which can be easily implemented in android or embedded linux. Both operating systems can be configured using ARM processor. The ARM A9 processor is thermally constrained and cost sensitive device which is ideal for green computing products.

The Low powered terminal devices are embedded with linux or android operating system. These operating system does not require frequent updating of operating system which makes our low powered device as a maintenance free device. Today computing users always want to do their operation efficiently and does not want to take care of the maintenance of the system. The fat client often requires updating operating system.

The Low powered device requires maximum 7.5Watts for its normal operation. The power consumption can be drastically reduced using this terminal. The Traditional fat client consumes upto $70 \mathrm{~W}$ for normal operation.

The low powered terminal device generates less heat during its operation and also its emit less carbon compared to fat clients. The cooling required to cool IT infrastructure is marginally reduced in this devices.

The cost of low powered client terminal is low comparing to traditional fat clients. This will help to reduce the cost of IT infrastructure.

\section{Physical Server.}

The physical server will be any physical fat client or xeon based machines. The hardware configuration can be selected based on the number of client going to access the services provided by server or Host pc.

The physical server will be served based machines supports multitasking environment. Server based machines are always ideal in multi user computing environment. The power consumption of xeon servers ranges from $150 \mathrm{~W}$ to $300 \mathrm{~W}$ for normal operation.

\section{E. Virtual Machine}

The virtual machine is implemented on physical server. We can use hyper visor or VM Ware vsphere suites. This software will divide the physical server into logical units. Initially we can use primary operating system as base operating system then we can use virtualization software to make the server into two logical units and the second operating system will be configured on logical unit. It can also be called as guest operating system.

\section{F. Implementing Private Clouds}

The private clouds are configured in physical operating system and guest operating system then using virtualization software like hypervisor and we can run multiple operating system simultaneously.

The Terminal client can now connect with any one of the operating system to use hosted services using embedded web browser.

The physical machine now runs both operating system simultaneously using virtualization software. The cloud services or hosted services are now running in both physical operating system and guest operating system.

The low powered client terminal is embedded with browser and support standard networking protocols. The cloud servers are now active on both servers and all clients can access both hosted services provided by servers.

\section{A. Support for HDMI Ports \& AV Ports}

\section{Features of our proposed system}

Our proposed system comes with HDMI \& AV Ports which enables our proposed system to connect with any LCD TVs or normal TV which makes no need for computer monitor in all places. In some places few client terminal user just require a fat client for purpose of monitoring few activities. In this case, using our 
proposed system one can easily change the existing TV into client terminal and watch the activities like normal pc, which enables more power saving \& low IT infrastructure cost.

B. Inbuilt application support

Proposed Client Terminal System have inbuilt application support which enables client user to do small task without the support of private cloud servers. Today android application markets have more than 1 lakh application which enables user to easily perform daily activities with the help of those applications.

C. Less Foot Print.

Proposed Client terminal System is designed using the embedded ARM Processor which makes the client terminal much smaller than the fat clients. This saves IT Infrastructure space. The small space can occupy more client terminal. This enables clean IT Infrastructure with less space. This will enables to create IT Infrastructure for more people in small space.

\section{Quiet Environment}

Proposed Client terminal is designed using the fan less processor which enables quiet environment. The fat client will produce a minimal noise due to mechanical components like CPU fan and hard disk access. In our environment the fan less design and DOM will not make any noise.

\section{Comparision}

Power Consumption : CPU vs Proposed Client

Fig.2.Power Consumption

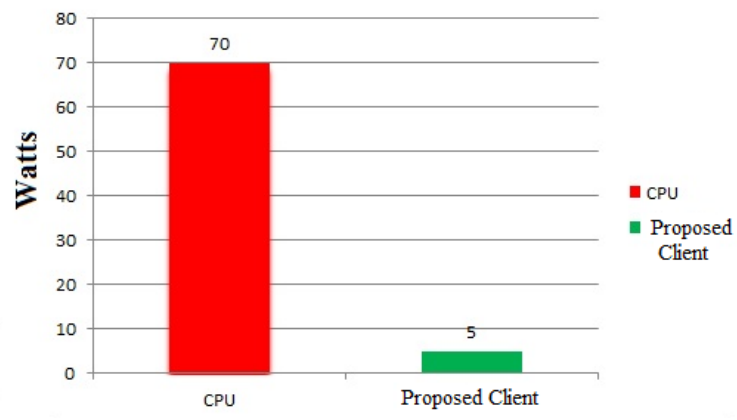

Licensing Cost : CPU vs Proposed Client.

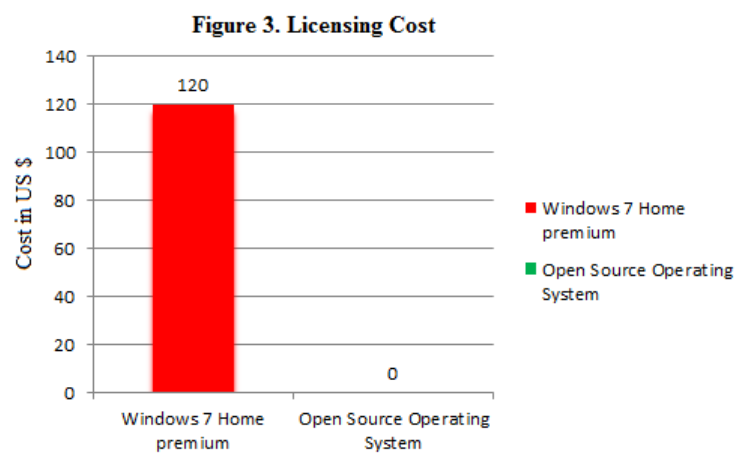

Cost of Hardware : CPU vs Proposed Client. 


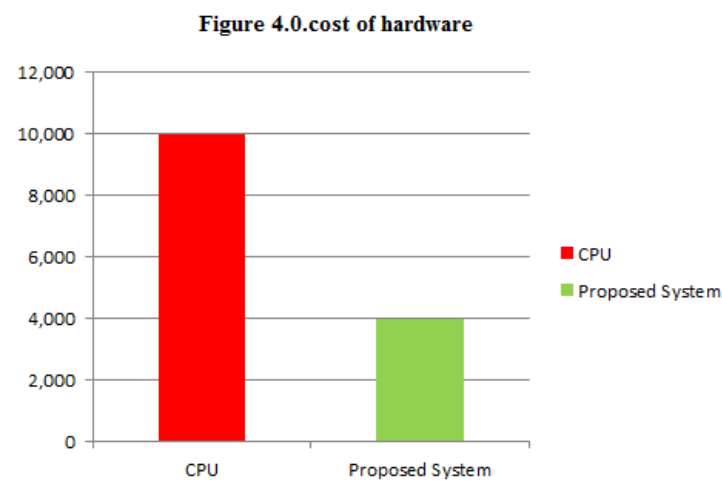

analysis

Our Proposed system is mainly designed to reduce the cost of IT Infrastructure set up \& operation. Low powered client device will enable to establish low cost IT infrastructure. The main draw backs in rural IT infrastructure are maintenance. Our proposed system will help to solve this problem. The maintenance of private cloud server is enough to run its other clients nodes. Remote firm ware update will enables it as $100 \%$ trouble free environment.

By analyzing the above charts and tables the proposed system will bring down the IT Infrastructure cost in terms of power consumption, licensing cost, space requirement and cost of physical device.

\section{Conclusion and future enchancement}

The proposed system will help to build the rural IT infrastructure at low cost. The rural education institutes can set up the IT infrastructure at low cost. Instead of going for fat clients, the proposed system will help to create more terminal for users.

The low power consumption enables using the proposed system in any geographical location where power is a big constraint for setting up IT infra-structure. The proposed system will bring down the E waste by using clients for long time without changing any hardware.

The proposed system does not presently support common operating system due to lack of hardware support. The future work will focus on improving the hardware support to support common operating system by staying within the same low power constraint and foot print.

\section{Acknowledgement}

A sincere gratitude expressed to the anonymous reviewers for providing some useful suggestions.

\section{References}

[1] Segal, I.. ""When Is Zero Client Not Zero Client?", SysGen, Inc"

[2] Mell, P. and Grance, T. (2011-09). "The NIST Definition of Cloud Computing. NIST Special Publication 800-145 (September 2011). National Institute of Standards and Technology, U.S. Department of Commerce"

[3] Sherbak, T., Sweere, N., and Belapurkar, V.. "Virtualized Enterprise Storage for Flexible, Scalable Private Clouds. Reprinted from Dell Power Solutions, 2012 Issue $1 "$

[4] Chou, Timothy. Introduction to Cloud Computing: Business \&Technology. http://www.scribd.com/doc/64699897/Introduction-toCloud-Computing-Business-and-Technology

[5] Wang, R."Tuesday's Tip: Understanding The Many Flavors ofCloudComputingandSaaS".

[6] Nieh, Jason; Novik, Naomi \&., Yang, S. Jae (December, 2005). "A Comparison of Thin-Client Computing Architectures". Technical Report CUCS-022-00 (New York: Network Computing Laboratory, Columbia University

[7] http://www.nomachine.com/documentation/pdf/cucs-022-00.pdf

[8] Madden, B. (May 19, 2012) (2010-05-19). "Wyse hopes to shake up the thin client industry with a new zero client platform. Will it work?"

[9] Greaves, J. (of Carpathia Hosting) and Potti, S. (of Citrix). Uploaded by CarpathiaHosting on Feb 22, 2010. "Flex-Tenancy: Secure Multi-Tenancy Network Environments"

[10] I.S. Jacobs and C.P. Bean, "Fine particles, thin films and exchange anisotropy," in Magnetism, vol. III, G.T. Rado and H. Suhl, Eds. New York: Academic, 1963, pp. 271-350.

[11] K. Elissa, "Title of paper if known," unpublished.

[12] R. Nicole, "Title of paper with only first word capitalized," J. Name Stand. Abbrev., in press.

[13] Y. Yorozu, M. Hirano, K. Oka, and Y. Tagawa, "Electron spectroscopy studies on magneto-optical media and plastic substrate interface," IEEE Transl. J. Magn. Japan, vol. 2, pp. 740-741, August 1987 [Digests 9th Annual Conf. Magnetics Japan, p. 301, 1982].

[14] M. Young, The Technical Writer's Handbook. Mill Valley, CA: University Science, 1989. 